\title{
A NECESSARY AND SUFFICIENT CONDITION FOR UNIQUENESS OF SOLUTIONS OF SINGULAR DIFFERENTIAL INEQUALITIES
}

\author{
ALAN V. LAIR \\ Departineut of Mathematics and Computer Science \\ Air Force Institute of Technology \\ Wright-Patterson AFB, OH 45433 \\ (Received December 12, 1988 and in revised form July 20, 1989)
}

ABSTRACT. The author proves that the abstract differential inequality $\left\|u^{\prime}(t)-A(t) u(t)\right\|^{2} \leq \gamma\left[\omega(t)+\int_{0}^{t} \omega(\eta) d \eta\right]$ in which the linear operator $A(t)-M(t)+$ $N(t), M$ symmetric and $N$ antisymmetric, is in general unbounded, $\omega(t)-t^{-2} \psi(t)\|u(t)\|^{2}$ $+\|M(t) u(t)\|\|u(t)\|$ and $\boldsymbol{\gamma}$ is a positive constant has a nontrivial solution near $t=0$ which vanishes at $t=0$ if and only if $\int_{0}^{1} t^{-1} \psi(t) d t=\infty$. The author also shows that the second order differential inequality $\left\|u^{\prime \prime}(t)-A(t) u(t)\right\|^{2} \leq \gamma\left[\mu(t)+\int_{0}^{t} \mu(\eta) d \eta\right]$ in which $\mu(t)-t^{-4} \psi_{0}(t)\|u(t)\|^{2}+t^{-2} \psi_{1}(t)\left\|u^{\prime}(t)\right\|^{2}$ has a nontrivial solution near $t-0$ such that $u(0)-u^{\prime}(0)=0$ if and only if either $\int_{0}^{1} t^{-1} \psi_{0}(t) d t=\infty$ or $\int_{0}^{1} t^{-1} \psi_{1}(t) d t=\infty$. Some mild restrictions are placed on the operators $M$ and $N$. These results extend earlier uniqueness theorems of Hile and Protter.

KEY WORDS AND PHRASES. Uniqueness of solution, singular differential inequality, singular equation.

1980 AMS SUBJECT CLASSIFICATION. 34G20, $34 \mathrm{G} 10$.

1. INTRODUCTION.

Let $\mathrm{H}$ be a complex Hilbert space with the usual inner product and norm notation and let $A$ be an linear, in general unbounded, operator defined on a non-trivial domain $D$ in $H$. Assuming the operator $A=M+N$ where $M$ is symmetric and $N$ is antisymmetric, we consider the differential inequalities

$$
\left\|u^{\prime}(t)-A(t) u(t)\right\|^{2} \leq \gamma\left[\omega(t)+\int_{0}^{t} \omega(\eta) d \eta\right]
$$


where $\omega(t)=\frac{\psi(t)}{t^{2}}\|u(t)\|^{2}+\|M(t) u(t)\|\|u(t)\|$ and

$$
\left\|u^{\prime \prime}(t)-A(t) u(t)\right\|^{2} \leq \gamma\left[\mu(t)+\int_{0}^{t} \mu(\eta) d \eta\right]
$$

where $\mu(t)=\frac{\psi_{0}(t)}{t^{4}}\|u(t)\|^{2}+\frac{\psi_{1}(t)}{t^{2}}\left\|u^{\prime}(t)\right\|^{2}$ and $\gamma$ is a positive constant. We show, under rather general conditions on $M$ and $N$, that a necessary and sufficient condition for the existence of an interval $(0, T]$ on which (1.1) will have a nontrivial solution vanishing at $t=0$ is

$$
\int_{0}^{1} \frac{\phi(t)}{t} d t=\infty .
$$

Furthermore, we show that a necessary and sufficient condition for the existence of an interval $(0, T)$ on which (1.2) will have a nontrivial solution vanishing at $t=0$ is either

or

$$
\int_{0}^{1} \frac{\psi_{0}(t)}{t} d t=\infty
$$

$$
\int_{0}^{1} \frac{\psi_{1}(t)}{t} d t=\infty .
$$

Our results extend those of Hile and Protter [1] who prove that the only solution of (1.1) and likewise for (1.2) with homogenous initial conditions is the trivial one provided the functions $t^{-2} \psi(t), t^{-4} \psi_{0}(t)$ and $t^{-2} \psi_{1}(t)$ are bounded. Thus our proofs of necessity (See Theorems 2 and 4.) contain the uniqueness theorems of [1] (See Theorems 1 and 3 of [1].) as a special case. Furthermore our results allow for less stringent requirements on the operators $M$ and $N$ in that certain kinds of singularities at t-0 are allowed. Also we show that our results are best in that (1.1) (or (1.2)) will have a nontrivial solution (with zero initial data) on some interval $(0, T]$ for $T$ small if $(1.3)$ (or $(1.4)$ or $(1.5)$ ) holds.

Other works considering singular equations abound. (See e.g. [2]-[11] and their references.) of particular relevance to our results here are [2], [3] and [4]. Lees and Protter [2] show, for A = M - a uniformly elliptic second order partial differential operator (in $x$ ), that a differential inequality similar to (1.1) has only the trivial solution vanishing at $t=0$ when $\psi$ is unity provided the $L_{2}$ norm (in $x$ ) of the spatial gradient of $u$ has an infinite order zero initially. Our work confirms the necessity of some such additional information on $u$ in order to obtain their uniqueness. Donaldson and Goldstein [3] and Ames [4] consider specific equations which are special cases of (1.1) and (1.2) and thus obtain sharper results. In particular, Donaldson and Goldstein [3] prove that the only solution of $u^{\prime}-A u-P(t) u$ vanishing initially is the trivial one provided $P(t)-(1 / t+b) I$, for some real $b$, is dissipative for all positive $t$ and the operator $A=-s^{2}$ where $S$ is self-adjoint and indpendent of $t$. They also show that for $P(t)=(1+\varepsilon) / t+b$, for any real $b$, nontrivial solutions exist. These results are, of course, consistent with ours. Indeed 
our results show that if $\psi$ is any positive constant, then (1.1) has a nontrivial solution near zero which vanishes at zero. (See Theorem 1.) They also consider the equation

$$
v^{\prime \prime}(t)+\alpha(t) v^{\prime}(t)=A v(t)
$$

which is the well known abstract Euler-Poisson-Darboux (EPD) equation if $\alpha(t)-k / t$, $k$ constant, and prove uniqueness for the initial value problem provided $\alpha(t) \geq-1 / t$. These results of Donald and Goldstein [3] have been extended by Goldstein [5] as well as Arrate and Garcia [6]. Ames [4] also considers (1.6) with $\alpha(t)=\psi(t) / t$ (where $\psi$ has properties somewhat similar to ours) but requires only that the operator $A$ be symmetric (and independent of $t$ ). Furthermore it is known that the solution to the EPD equation (A - the Laplacian) is not unique if $k<0$ (See e.g., [4].). These results are again consistent with ours. Indeed, for $\alpha(t)-k / t$ corresponds to taking $\psi_{1}=1, \psi_{0}=0$ in $(1.2)$ and hence (1.4) holds implying a nontrivial solution exists near zero (See Theorem 3.).

We note that the form of the function $\alpha$ in [4] along with the work of Hile and Protter [1] and Garofalo [7] have been the major motivating factors in this study and especially choosing the form of $\omega$ in (1.1) and of $\mu$ in (1.2). Finally we note that the extension of the uniqueness theorems of [1] to the $n^{\text {th }}$ order time derivative case with $A$ independent of $t$ is contained in [12].

2. THE FIRST ORDER CASE.

Throughtout this section we assume $\psi \in C^{2}((0, \infty))$ satisfying

$$
\psi>0, \psi^{\prime} \geq 0, \psi^{\prime \prime} \leq 0 \text {. }
$$

Consequently the function $\psi(t) / t$ is nonincreasing and hence

$$
t \psi^{\prime}(t) \leq \psi(t) \text {. }
$$

We now give assumptions on the linear operator $A$ which, except for (iii) and (iv), match those of [1] while (iii) and (iv) are more general than the similar conditions given in [1]. It should be noted that not all of these will be needed in the proof of sufficiency.

For $t_{0}>0$, let $c^{\star}\left(\left[0, t_{0}\right] ; D\right)$ be the set of $u \in c\left(\left[0, t_{0}\right] ; D\right) \cap c^{1}\left(\left(0, t_{0}\right] ; H\right)$ such that $\left\|u^{\prime}(t)\right\|$ is bounded on $\left(0, t_{0}\right)$.

Condition ( $I)$. We assume there exists $T>0$ so that the linear operator $A(t)$, with nontrivial domain $D(i . e ., D \neq(0))$, satisfies the following:

(i) $\quad A(t)=M(t)+N(t), M$ is symmetric and $N$ is antisymmetric;

(ii) For each $u \in C^{*}([0, T] ; D)$, the functions $M(t) u(t)$ and $N(t) u(t)$ are bounded and continuous on $(0, \mathrm{~T}]$;

(iii) There exists a positive constant $\gamma_{1}$ such that for all weD and $t \in(0, \Upsilon]$ $\operatorname{Re}(M(t) w, N(t) w) \geq-\gamma_{1}\left[\|M(t) w\|\|w\|+\frac{\psi(t)}{t^{2}}\|w\|^{2}\right]$.

(iv) For each $u \in C^{*}([0, T] ; D)$ satisfying $(1.1)$, the function $(M(t) u(t), u(t))$ is continuously differentiable on $(0, T]$ and there exists a positive constant $\gamma_{2}$ such that for all $t \epsilon(0, T]$

$d / d t(M(t) u(t), u(t))-2 \operatorname{Re}\left(M(t) u(t), u^{\prime}(t)\right)$

$$
\geq-\gamma_{2}\left[\|M(t) u(t)\|\|u(t)\|+\frac{\psi(t)}{t^{2}}\|u(t)\|^{2}\right] \text {. }
$$


Sufficiency. Although the proof of necessity will require that the operator $A$ satsify condition (I), sufficiency will not require properties (iii) and (iv).

Furthermore, we show that the nontrivial function satisfying (1.1) actually satisfies a much sharper inequality (See (2.5) below.) than (1.1).

THEOREM 1. (Sufficiency) Suppose (1.3) holds and the operator A satisfies condition ( $I$ ) except possibly for parts (iii) and (iv). Then there exists a $T>0$ such that inequality ( 1.1$)$ has a nontrivial solution on $(0, T]$ contained in $C^{*}([0, T] ; D)$ which vanishes at $t=0$.

PROOF. Let $v$ be any nonzero element of $D$. Since (1.3) holds and the function $\psi(t) / t$ is nondecreasing, we have $\lim \psi(t) / t=\infty$. Combining this result with part (ii) of condition (I) yields

$$
\lim _{t \downarrow 0} \psi(t) t^{-2}\left[1+\|A(t) v\|^{2}\right]^{-1}=\infty
$$

and thus we may choose $T \epsilon(0, T]$ so that $\gamma \psi(t) / t^{2} \geq 2\left[1+\|A(t) v\|^{2}\right]\|v\|^{-2}$ for all $t \epsilon$ $(0, T]$ where $\gamma$ comes from (1.1). Define $K=\sup (\|A(t) v\|: 0<t \leq T)$ which is finite because of condition (I). Then $\gamma \psi(t) / t^{2} \geq 2\left[1+\|A(t) v\|^{2}\right]\|v\|^{-2}$ for all $t \in(0, T]$ and we define

$$
\xi(t)=\int_{t}^{\mathrm{T}}\left[(\gamma / 2) \eta^{-2} \psi(\eta)-\mathrm{K}^{2}\|\mathrm{v}\|^{-2}\right]^{1 / 2} \mathrm{~d} \eta \quad, \quad 0<\mathrm{t} \leq \mathrm{T}
$$

Let $u(t)=e^{-\xi(t)} v$. We need to show

$$
\lim _{t \downarrow 0} u(t)=0
$$

and that $u$ satisfies $(1.1)$ on $(0, T]$. To determine the initial value of $u$, note that since $\psi$ is nondecreasing, $\lim _{t \downarrow 0} \psi(t)$ exists. Let $\lim _{t \downarrow 0} \phi(t)=L, 0 \leq L<\infty$. If $L=0$, then $\psi^{1 / 2} \geq \psi$ near zero and thus (1.3) implies $\int_{0}^{T} t^{-1}[\psi(t)]^{1 / 2} d t=\infty$ and hence $\xi(t) \rightarrow$ $\infty$ as $t \downarrow 0$ which in turn yields (2.3). On the other hand, if $L \neq 0$, it is clear that $\xi(t) \rightarrow \infty$ as $t+0$ and thus (2.3) holds.

To show that $u$ satisfies $(1.1)$ on $(0, T]$, note that straightforward calculations give

$$
\begin{aligned}
\| u^{\prime}(t)- & A(t) u(t)\left\|^{2} \leq 2\right\| u^{\prime}(t)\left\|^{2}+2\right\| A(t) u(t) \|^{2} \\
& -2\left[(\gamma / 2) t^{-2} \psi(t)-k^{2}\|v\|^{-2}\right] e^{-2 \xi(t)}\|v\|^{2}+2 e^{-2 \xi(t)}\|A(t) v\|^{2}
\end{aligned}
$$

Since $\|A(t) v\| \leq K$, inequality (2.4) implies

$$
\left\|u^{\prime}(t)-A(t) u(t)\right\|^{2} \leq 2\left[(\gamma / 2) t^{-2} \psi(t)\|v\|^{2}\right] e^{-2 \xi(t)}-\gamma t^{-2} \psi(t)\|u\|^{2}
$$

and thus (1.1) holds. This completes the proof.

Necessity. Suppose

$$
\int_{0}^{1} \frac{\psi(t)}{t} d t<\infty .
$$

Then the monotonicity of $\psi$ gives $\lim \psi(t)-0$. Also, without loss of generality, we may assume $\lim \phi(t) / t=\infty$. Indeed $\lim \psi(t) / t$ exists (possibly infinite) since $\psi(t) / t$ is nonincreasing; and furthermore, if $\lim \psi(t) / t<\infty$, inequality (1.1) is still valid 
on $(0, T]$ if $\psi(t)$ is replaced with $C t^{1 / 2}$ for a sufficiently large constant $C$ (depending only on $T$ ) and hence $\lim _{t \downarrow 0} \psi(t) / t=\infty$. Additionally, as a consequence of (2.6) and the monotoncity of $\psi(t) / t$, we have

$$
\begin{aligned}
& t^{k} \int_{t}^{T} \eta^{-k-1} \psi(\eta) d \eta \leq t^{k}\left[t^{-1} \psi(t)\right] \int_{t}^{T} \eta^{-k} d \eta-t^{k-1} \psi(t)\left(-T^{-k+1}+t^{-k+1}\right) /(k-1) \\
& \leq \psi(t) /(k-1) \quad \text { for any } 0<t \leq T, k>1 \text {, }
\end{aligned}
$$

and hence

$$
t^{k} \int_{t}^{T} \eta-k-1 \psi(\eta) d \eta \leq \psi(t) /(k-1), k>1,0<t \leq T .
$$

Before proving necessity (Theorem 2), we need some preliminary lemmas.

LEMMA 1. Suppose $\phi$ satisfies (2.6). Let $\rho(t)=\psi(t) / t^{2}, \lambda(t)-\int_{0}^{t} \psi(\eta) / \eta d \eta$, and suppose $h$ and $r$ are nonnegative functions continuous on $(0, \Upsilon]$ for some $T>0$. Furthermore, assume $r(t)$ and $h(t) / t$ are bounded near zero. Then, for all $\varepsilon>0$ and all $\mathrm{T} \in[0, \mathrm{~T}]$, we have

$$
2 \int_{0}^{\mathrm{T}} \rho(\xi) \int_{0}^{\xi} \mathrm{h}(\eta) \mathrm{r}(\eta) \mathrm{d} \eta \leq \varepsilon \int_{0}^{\mathrm{T}} \rho(\eta) \mathrm{h}^{2}(\eta) \mathrm{d} \eta+\varepsilon^{-1} \lambda(\mathrm{T}) \int_{0}^{\mathrm{T}}(\mathrm{r}(\eta))^{2} \mathrm{~d} \eta
$$

PROOF. Since the result is trivial for $\mathrm{T}=0$, we consider only the case $\mathrm{T}>0$. Thus suppose $0<t<T$ and use Cauchy-Schwarz along with elementary estimates to $\operatorname{get}\left(\Psi(t)=\int_{0}^{t}[\rho(\eta)]^{-1}[r(\eta)]^{2} \mathrm{~d} \eta\right)$

$$
\begin{aligned}
& 2 \int_{0}^{t} \rho(\eta) \int_{0}^{\eta} h(s) r(s) s d \eta=2 \int_{0}^{t} \rho(\eta) \int_{0}^{\eta}[\rho(s)]^{1 / 2} h(s)[\rho(s)]^{-1 / 2} r(s) \mathrm{d} s \mathrm{~d} \eta \\
& \leq 2 \int_{0}^{t} \rho(\eta)\left[\int_{0}^{\eta} \rho \mathrm{h}^{2} \mathrm{ds}\right]^{1 / 2}[\Psi(\eta)]^{1 / 2} \mathrm{~d} \eta \leq 2\left[\int_{0}^{t} \rho \mathrm{h}^{2} \mathrm{ds}\right]^{1 / 2} \int_{0}^{\mathrm{t}} \rho(\eta)[\Psi(\eta)]^{1 / 2} \mathrm{~d} \eta \\
& \leq \varepsilon \int_{0}^{t} \rho \mathrm{h}^{2} \mathrm{ds}+\varepsilon^{-1}\left[\int_{0}^{t} \rho(\eta)[\Psi(\eta)]^{1 / 2} \mathrm{~d} \eta\right]^{2} \text {. }
\end{aligned}
$$

The last integral in (2.9) admits the estimate

$$
\begin{aligned}
{\left[\int_{0}^{t} \rho(\eta)[\Psi(\eta)]^{1 / 2} \mathrm{~d} \eta\right]^{2} } & \leq\left[\int_{0}^{t} \eta^{1 / 2}[\rho(\eta)]^{1 / 2} \eta^{-1 / 2}[\rho(\eta)]^{1 / 2}[\Psi(\eta)]^{1 / 2} \mathrm{~d} \eta\right]^{2} \\
& \leq\left[\int_{0}^{t} \eta \rho(\eta) \mathrm{d} \eta\right]\left[\int_{0}^{t} \eta^{-1} \rho(\eta) \Psi(\eta) \mathrm{d} \eta\right]-\lambda(t) \int_{0}^{t} \mathrm{R}^{\prime}(\eta) \Psi(\eta) \mathrm{d} \eta
\end{aligned}
$$

where $R(t) \equiv-\int_{t}^{T} \eta^{-1} \rho(\eta) \mathrm{d} \eta$ for $t<\mathrm{T}$. Since

$$
0 \leq-R(\eta) \Psi(\eta) \leq\left[\int_{t}^{\mathrm{T}} \eta^{-1} \psi(\eta) \mathrm{d} \eta\right]\left[t^{-2} \int_{0}^{\mathrm{t}}[\rho(\eta)]^{-1} \mathrm{r}^{2}(\eta) \mathrm{d} \eta\right]
$$

and application of L'Hospital's rule gives 


$$
\begin{aligned}
\lim _{t \downarrow 0} t^{-2} \int_{0}^{t}[\rho(\eta)]^{-1} r^{2}(\eta) d \eta & =\lim _{t \downarrow 0} \frac{\int_{0}^{t} \eta^{2}[\psi(\eta)]^{-1} r^{2}(\eta) d \eta}{t^{2}} \\
& -(1 / 2) \prod_{t+0} \lim _{t+0} r^{2}(t) t / \psi(t)-0
\end{aligned}
$$

where the last equality holds because $r$ is bounded near zero and $\psi(t) / t \rightarrow \infty$, we get $\lim R(\eta) \Psi(\eta)=0$. Using this result, we integrate by parts in the last integral in $\eta \downarrow 0$

(2.10) and obtain

$$
\left[\int_{0}^{t} \rho(\eta)[\Psi(\eta)]^{1 / 2} \mathrm{~d} \eta\right]^{2} \leq \lambda(t)\left[R(t) \Psi(t)-\int_{0}^{t} R(\eta) \Psi^{\prime}(\eta) \mathrm{d} \eta\right]
$$

Since $\lambda(t)$ and $\Psi(t)$ are nonnegative while $R(t)$ is nonpositive, we may discard the first expression on the right side of (2.11). Also (2.7) with $k-2$ gives exactly $-R(\eta)[\rho(\eta)]^{-1} \leq 1$ so that $-R(\eta) \Psi^{\prime}(\eta) \leq r^{2}(\eta)$. Substitution of this into (2.11) and the resulting inequality into (2.9) yields (2.8). This completes the proof.

LEMMA 2. Suppose $z \in C^{*}([0, T] ; D)$ such that $z(0)-0$. Then

$$
\int_{0}^{t} \rho(\eta)\|z(\eta)\|^{2} \mathrm{~d} \eta \leq 4 \lambda(t) \int_{0}^{t}\left\|z^{\prime}(\eta)-N(\eta) z(\eta)\right\|^{2} \mathrm{~d} \eta
$$

where the functions $\rho$ and $\lambda$ are given in Lemma 1 .

PROOF. Since $z(0)=0$ and the operator $N$ is antisymmetric, we get

$$
\|z(\eta)\|^{2}=2 \operatorname{Re} \int_{0}^{\eta}\left(z(s), z^{\prime}(s)-N(s) z(s)\right) d s \leq 2 \int_{0}^{\eta}\|z(s)\|\left\|z^{\prime}(s)-N(s) z(s)\right\| d s .
$$

Now multiply $(2.13)$ by $\rho(\eta)$, integrate over $[0, t]$ and apply inequality (2.8) to the resulting right side to get

$$
\begin{aligned}
\int_{0}^{t} \rho(\eta)\|z(\eta)\|^{2} \mathrm{~d} \eta & \leq 2 \int_{0}^{t} \rho(\eta) \int_{0}^{\eta}\|z(s)\|\left\|z^{\prime}(s)-N(s) z(s)\right\| \mathrm{d} s \mathrm{~d} \eta \\
& \leq \varepsilon \int_{0}^{t} \rho(\eta)\|\mathrm{z}(\eta)\|^{2} \mathrm{~d} \eta+\varepsilon^{-1} \lambda(\mathrm{t}) \int_{0}^{t}\left\|\mathrm{z}^{\prime}(\mathrm{s})-\mathrm{N}(\mathrm{s}) \mathrm{z}(\mathrm{s})\right\|^{2} \mathrm{~d} \eta .
\end{aligned}
$$

Taking $\varepsilon=1 / 2$ in this expression and simplifying yields (2.12). This completes the proof.

LEMMA 3. Suppose $0<T<\min (1, T)$ and $t_{0}>0$ is such that $t_{0}+T<1$. Also suppose the operator A satisfies condition (I) and $L u=u^{\prime}-A u$. Assume that $u \in$ $C^{*}([0, T] ; D)$ and $u(0)-u(T)-0$. Then, for all sufficiently large $\beta>0$, the size depending only on the constants $\gamma_{1}$ and $\gamma_{2}$ from condition (I), the following holds

$$
\beta^{2} \int_{0}^{\mathrm{T}} \tau^{-\beta-2} \mathrm{e}^{2 \tau^{-\beta}}\|\mathrm{u}\|^{2} \mathrm{dt}+\mathrm{C}_{0}[\lambda(\mathrm{T})]^{-1} \int_{0}^{\mathrm{T}} \rho \mathrm{e}^{2 \tau^{-\beta}}\|\mathrm{u}\|^{2} \mathrm{dt}+\mathrm{C}_{1} \int_{0}^{\mathrm{T}} \tau^{\beta} \mathrm{e}^{2 \tau^{-\beta}}\|\mathrm{Mu}\|^{2} \mathrm{dt} \leq \mathrm{C}_{2} \int_{0}^{\mathrm{T}} \mathrm{e}^{2 \tau^{-\beta}}\|\mathrm{Lu}\|^{2} \mathrm{dt}
$$

where $r=t+t_{0}, \rho(t)=t^{-2} \psi(t)$ and $C_{0}, C_{1}$ and $C_{2}$ are absolute constants.

PROOF. Following $[1, \mathrm{p} .61]$, we set $\varphi(t)=-\left(t+t_{0}\right)^{-\beta}$ and $\operatorname{define} v=\mathrm{e}^{-\varphi_{u}}$. Then $\mathrm{Lu}=\mathrm{e}^{\varphi}\left[\mathrm{v}^{\prime}+\varphi^{\prime} \mathrm{v}-\mathrm{Mv}-\mathrm{Nv}\right]$, and defining the function $\alpha$ (See $\left.[1, \mathrm{p} .62].\right)$ by $\alpha(t)-\mathrm{k}_{0}{ }^{\beta}$, 
we have $e^{-2 \varphi}\|\mathrm{Lu}\|^{2}=\left\|v^{\prime}+\varphi^{\prime} v-\alpha M v-(1-\alpha) M v-N v\right\|^{2}$. Thus, integrating with respect to $t$ from 0 to $T$, we get

$$
\begin{aligned}
\int \mathrm{e}^{-2 \varphi}\|\mathrm{Lu}\|^{2} \geq & 2 \operatorname{Re} \int\left(\mathrm{v}^{\prime}-\alpha M v-N v, \varphi^{\prime} \mathrm{v}-(1-\alpha) M v\right)+\int\left\|v^{\prime}-\alpha M v-N v\right\|^{2} \\
& =2 \operatorname{Re} \int \varphi^{\prime}\left(\mathrm{v}^{\prime}, \mathrm{v}\right)+2 \int \alpha(1-\alpha)\|\mathrm{Mv}\|^{2}-2 \int \alpha \varphi^{\prime}(\mathrm{Mv}, \mathrm{v})-2 \operatorname{Re} \int\left(\mathrm{v}^{\prime}, \mathrm{Mv}\right) \\
& +2 \operatorname{Re} \int(\mathrm{Nv}, \mathrm{Mv})+\int\left\|v^{\prime}-\mathrm{Nv}\right\|^{2} \\
& =\mathrm{I}_{1}+\ldots+\mathrm{I}_{6} .
\end{aligned}
$$

Using estimates for $I_{1}$ through $I_{3}$ identical to those in [1, proof of Lemma 1] and estimates virtually identical to those of $\mathrm{I}_{4}$ and $\mathrm{I}_{5}$ in the same lemma (the only difference is the $1-\alpha$ in [1] is replaced with 1 here) and using (2.12) above to estimate $I_{6}$ gives (2.14) and the proof is complete.

We may now prove necessity. It should be noted that Theorem 2 contains the results of [1; Theorem 1] as a special case.

THEOREM 2. (Necessity) Suppose the operator A satisfies condition (I) and there exists $T \in(0, T]$ such that $u \in C^{*}([0, T] ; D)$ is a solution of $(1.1)$ on $(0, T]$ with $u(0)$ - 0 . If the function $\psi$ satisfies (2.6), then $u=0$ on $[0, T)$.

PROOF. Following [1], we show that $u=0$ on $\left[0, T^{\prime}\right]$ for sufficiently small $T^{\prime}$. Once this has been done, we may then apply the results of [1, Theorem 1] on the interval $\left[\mathrm{T}^{\prime}, \mathrm{T}\right]$ where $\psi(t) / t^{2}$ is bounded to get $u=0$ on $[0, T]$. We choose $T^{\prime}$ less than one in such a way that $\lambda\left(T^{\prime}\right)^{-1}$ is large depending only on known constants (See inequality (2.15) below.) where the function $\lambda$ is defined in Lemma 1 and by hypothesis $\lambda(t) \downarrow 0$ as $t \downarrow 0$.

Let $\varepsilon>0$ be given and define the $c^{\infty}$ function 5 such that $5(t)-1$ for $0 \leq t \leq$ $T^{\prime}-\varepsilon,-0$ for $t \geq T^{\prime}$ and such that $0<\zeta<1$ for $T^{\prime}-\varepsilon<t<T^{\prime}$. The proof now proceeds as with [1]. (See inequality (2.6) of [1] and note that their $T_{0}$ is my $T^{\prime}$.) Applying Lemma 3 to $5 \mathrm{u}$ we get

$$
\begin{gathered}
\beta^{2} \int_{0}^{T^{\prime}-\varepsilon} \tau^{-\beta-2} e^{2 \tau^{-\beta}}\|\mathrm{u}\|^{2} \mathrm{dt}+\mathrm{C}_{0}\left[\lambda\left(\mathrm{T}^{\prime}\right)\right]^{-1} \int_{0}^{\mathrm{T}^{\prime}-\varepsilon} \rho \mathrm{e}^{2 \tau^{-\beta}}\|\mathrm{u}\|^{2} \mathrm{dt}+\mathrm{C}_{1} \int_{0}^{\mathrm{T}^{\prime}-\varepsilon}{ }^{-\beta} \mathrm{e}^{2 \tau^{-\beta}}\|\mathrm{Mu}\|^{2} \mathrm{dt} \\
\leq \mathrm{C}_{2} \int_{0}^{\mathrm{T}^{\prime}-\varepsilon} \mathrm{e}^{2 \tau^{-\beta}}\|\mathrm{Lu}\|^{2} \mathrm{dt}+\mathrm{C}_{2} \int_{\mathrm{T}^{\prime}-\varepsilon}^{\mathrm{T}} \mathrm{e}^{2 \tau^{-\beta}}\|\mathrm{L}(\zeta \mathrm{u})\|^{2} \mathrm{dt} .
\end{gathered}
$$

Using nearly identical arguments as in [1] we get, for arbitrary $k_{2}>0$,

$$
\begin{aligned}
\int_{0}^{\mathrm{T}} \mathrm{e}^{-\varepsilon} \mathrm{e}^{-\beta}\|\mathrm{Lu}\|^{2} \mathrm{dt} & \leq \mathrm{k} \int_{0} \int_{0}^{\mathrm{T}^{\prime}-\varepsilon} \mathrm{e}^{2 \tau^{-\beta}}{ }_{\tau}^{\beta+1}\|\mathrm{M}(\mathrm{t}) \mathrm{u}(\mathrm{t})\|^{2} \mathrm{dt} \\
& +\int_{0}^{\mathrm{T}^{\prime}-\varepsilon} \mathrm{e}^{2 \tau^{-\beta}}\left[2 \mathrm{c}(1+\rho)+\left(\mathrm{k}_{2}\right)^{-1} \tau_{\tau}^{-\beta-1} \mathrm{c}^{2}\right]\|\mathrm{u}(\mathrm{t})\|^{2} \mathrm{dt} .
\end{aligned}
$$

Hence, by choosing $k_{2}$ sufficiently small (depending only on $C_{1}$ and $C_{2}$ ), $\beta$ sufficiently large (depending only on $t_{0}, \gamma$ and $k_{2}$ (and hence $C_{1}$ and $C_{2}$ ) and $T$ ' sufficiently small (so that $\lambda\left(T^{\prime}\right)^{-1}>2 C_{2} \gamma\left(\rho(t)^{-1}+1\right) / C_{0}$ for $0<t<T$ ), and doing more estimates as in [1], we get

$$
\beta^{2} \int_{0}^{T^{\prime}-\varepsilon}\|u\|^{2} d t \leq 2 C_{2} \int_{T^{\prime}-\varepsilon}^{T^{\prime}}\|L(\zeta u)\|^{2} d t .
$$


Letting $\beta \rightarrow \infty$, we get $u=0$ on $\left[0, T^{\prime}-\varepsilon\right]$ and hence on $\left[0, T^{\prime}\right]$. This completes the proof.

3. THE SECOND ORDER CASE.

Throughout this section we assume $\psi_{i} \in C^{2}((0, \infty)), \quad i=0,1$, and

$$
\boldsymbol{\psi}_{i}>0, \boldsymbol{\psi}_{i}^{\prime} \geq 0, \boldsymbol{\psi}_{i}^{\prime \prime} \leq 0 \quad \text { on }(0, \infty), i=0,1 \text {. }
$$

Consequently the functions $\psi_{i}(t) / t$ are nonincreasing and hence

$$
t \psi_{i}^{\prime}(t) \leq \psi_{i}(t) \quad \text { on }(0, \infty), i=0,1 \text {. }
$$

We now give assumptions on the operator A which, except for (iii), match those of [1] while (iii) is more general than the similar conditions in [1] in that here the coefficients need not be bounded.

For $t_{0}>0$, let $c_{*}\left(\left[0, t_{0}\right] ; D\right)$ be the set of $u \in c\left(\left[0, t_{0}\right] ; D\right) \cap c^{1}\left(\left[0, t_{0}\right] ; H\right) \cap$ $C^{2}\left(\left(0, t_{0}\right] ; H\right)$ such that $\left\|u^{\prime \prime}(t)\right\|$ is bounded on $\left(0, t_{0}\right]$.

Condition (II). We assume there exists $T>0$ such that the linear operator

$A(t)$, with nontrivial domain $D$ (i.e., $D \neq(0)$ ), satisfies the following:

(i) $\quad A(t)=M(t)+N(t), M$ is symmetric and $N$ is antisymmetric;

(ii) For each $u \in C_{*}([0, \Upsilon] ; D)$, the functions $M(t) u(t)$ and $N(t) u(t)$ are bounded and continuous on $(0, T]$;

(iii) For nonnegative constant $\gamma_{3}$, we let $F(t)=\gamma_{3}\left[\frac{\psi_{0}(t)}{t^{3}}\|u(t)\|^{2}+\frac{\psi_{1}(t)}{t}\left\|u^{\prime}(t)\right\|^{2}\right]$.

For funtions $u \in C_{*}([0, T] ; D)$, we assume the functions $\operatorname{Re}\left(N(t) u(t), u^{\prime}(t)\right)$ and $(M(t) u(t), u(t))$ are continuously differentiable on $(0, \Upsilon]$ and satisfy the following on $(0, T]$ :

$$
\begin{aligned}
& (d / d t) \operatorname{Re}\left(N(t) u(t), u^{\prime}(t)\right)-\operatorname{Re}\left(N(t) u(t), u^{\prime \prime}(t)\right) \geq-F(t) \\
& (d / d t)(M(t) u(t), u(t))-2 \operatorname{Re}\left(M(t) u(t), u^{\prime}(t)\right) \geq-F(t) \\
& \operatorname{Re}(M(t) u(t), N(t) u(t)) \geq-F(t) .
\end{aligned}
$$

Sufficiency. Not all of Condition (II) will be needed to prove sufficiency, and as in the the first order case, we show that our solution actually satisfies a much sharper estimate than (1.2). (See inequalities (3.4) and (3.10).) However, before proving sufficiency, we need a preliminary result.

LEMMA 4. Let $\phi(t)=\min \left(\psi_{0}(t), C\right)$ where $C$ is any positive number and suppose (1.4) holds. The function $\phi(t) / t$ is nonincreasing on $(0, \infty)$ and

$$
\int_{0}^{1} \phi(t) / t d t=\infty .
$$

PROOF. Clearly $\phi(t) / t$ is nonincreasing since $\psi_{0}$ (See inequality (3.2).) has that same property. To prove (3.3), we shall assume, without loss of generality, that there exists a decreasing sequence of numbers $\left\{a_{n}\right\}$ in the open interval $(0,1)$ converging to zero such that $\phi\left(a_{n}\right)=C-\psi_{0}\left(a_{n}\right), n=1,2, \ldots$ If this were not the case, it must be that $\phi=\psi_{0}<C$ near zero or $\phi=C<\psi_{0}$ near 0 and in either case the result would hold trivially. Choose a subsequence $\left\{a_{n_{j}}\right\}$ of $\left\{a_{n}\right\}$ such that $a_{n_{1}}=$ $a_{1}$, and $2 a_{n_{j+1}} \leq a_{n_{j}}$ for all $j$. Since $\phi(t) / t$ is nonincreasing and $\phi\left(a_{n}\right) / a_{n}-c / a_{n}$, we 
get

$$
\begin{aligned}
\int_{0}^{1} \phi(t) / t d t & -\sum_{n=1}^{\infty} \int_{a_{n+1}}^{a} \phi(t) / t d t-\sum_{j=1}^{\infty} \int_{a_{n_{j}+1}}^{a} \phi(t) / t d t \\
& \geq \sum_{j=1}^{\infty} \int_{a_{n_{j+1}}}^{n_{j}} \phi\left(a_{n_{j}}\right) / a_{n_{j}} d t-\sum_{j=1}^{\infty} c\left[1-a_{n_{j+1}} / a_{n_{j}}\right] \geq \sum_{j=1}^{\infty} c / 2-\infty .
\end{aligned}
$$

This completes the proof.

THEOREM 3. (Sufficiency) Suppose that either (1.4) or (1.5) holds and the operator A satisfies condition (II) except possibly for part (iii). Then there exists $\mathrm{T}>0$ such that inequality (1.2) has a nontrivial solution on $(0, T]$ contained in $C_{\star}([0, T] ; D)$ which vanishes at $t=0$.

PROOF. Suppose (1.5) holds and let $\mathrm{v}$ be any nonzero element of D. Using the function $\psi_{1}$ in place the function $\psi$ in the proof of Theorem 1 , choose the constants $\mathrm{K}$ and $T$ and the function $\xi$ as in the proof of Theorem 1. (In addition, we must have $T \leq$ 1.) Using analysis similar to that of the first order case, it is easy to show that the function $u(t)=\left[\int_{0}^{t} e^{-\xi(s)} d s\right]$ v satisfies $\left\|u^{\prime \prime}(t)-A(t) u(t)\right\|^{2} \leq \frac{\gamma \psi_{1}(t)}{t^{2}}\left\|u^{\prime}(t)\right\|^{2}$ on $(0, T]$ with $u(0)-u^{\prime}(0)-0$. Hence $u$ satisfies (1.2) and vanishes along with its first derivative at $t=0$.

Now suppose (1.4) is satisfied. We shall find $T>0$ and function $u(t)$ which is a nontrivial solution of

$$
\begin{aligned}
& \left\|u^{\prime \prime}(t)-A(t) u(t)\right\|^{2} \leq \frac{\gamma \phi(t)}{t^{4}}\|u(t)\|^{2} \text { on }(0, T] \\
& u(0)-u^{\prime}(0)=0 .
\end{aligned}
$$

where $\phi(t)=\min \left(\psi_{0}(t), 8 / \gamma\right)$. Thus $u$ will also be a nontrivial solution of (1.2) since $\phi \leq \psi_{0}$. Let $\mathrm{v}$ be any nonzero element of $\mathrm{D}$. Since (1.4) holds and hence (3.3) holds (for $\mathrm{C}-8 / \gamma$ ), we may, in a manner similar to that in the proof of Theorem 1 , choose $0<\mathrm{T}_{0}<\mathrm{T}$ so that $\phi(t) / \mathrm{t}^{2} \geq(8 / \gamma)\left[1+\|\mathrm{A}(\mathrm{t}) \mathrm{v}\|^{2}\right]\|\mathrm{v}\|^{-2}$ for all $t \epsilon\left(0, \mathrm{~T}_{0}\right]$ where $\gamma$ comes from (1.2). Define $K=\sup \left(\|A(t) v\|: 0<t \leq T_{0}\right)$ which is finite because of condition (II). Then $(\gamma / 8) t^{-2} \phi(t)-k^{2}\|v\|^{-2}$ is nonnegative on $\left(0, T_{0}\right]$ and we define

$$
\xi(t)=\int_{t}^{\mathrm{T}}\left[(\gamma / 8) \eta^{-2} \phi(\eta)-\mathrm{k}^{2}\|\mathrm{v}\|^{-2}\right]^{1 / 2} \mathrm{~d} \eta .
$$

Before defining $T$ and $u$, we make some observations concerning the function $\xi$. As a result of (3.3) and the boundedness of $\phi$, we have $\int_{0}^{1} t^{-1}[\phi(t)]^{1 / 2} d t-\infty$.

Thus $\lim _{t \downarrow 0} \xi(t)=\infty$ and $\lim _{t \downarrow 0} \phi(t) / t=\infty$. Using L'Hospital's Rule, it is easy to show $\lim _{t \downarrow 0} e^{\xi(t)} \int_{0}^{t} e^{-\xi(s)} d s=0$. Hence we may choose $T \epsilon\left(0, T_{0}\right]$ so that 


$$
e^{-\xi(t)} \geq \int_{0}^{t} e^{-\xi(s)} d s \quad \text { for all } t \epsilon[0, T]
$$

Furthermore, if we define the function $S$ by $S(t)=t e^{-\xi(t)}-2 \int_{0}^{t} e^{-\xi(s)} d s$, then $S^{\prime}(t)=$ $\left\{\left[\gamma \phi(t) / 8-k^{2}\|v\|^{-2} t^{2}\right\}^{1 / 2}-1\right\} e^{-\xi(t)}$ so that $S^{\prime}(t) \leq 0$ on $\left(0, T_{0}\right]$ since $\phi \leq 8 / \gamma$. Thus since $\lim _{t \downarrow 0} S(t)=0$, we have $S(t) \leq 0$ on $\left(0, T_{0}\right]$ and hence on $(0, T]$. That is,

$$
2 \int_{0}^{t} e^{-\xi(s)} d s \geq t e^{-\xi(t)} \text { for all } t \in[0, T]
$$

We now let $u(t)=\left[\int_{0}^{t} e^{-\xi(s)} d s\right] v$ for $t \epsilon[0, T]$ and show that $u$, which is obviously nontrivial, satisfies (3.4), and hence also satisfies (1.2) and (3.5). Clearly $u(0)$ $=0$ and $u^{\prime}(0)=0$ since $\lim \xi(t)=\infty$. To show that $(3.4)$ holds, notice that on $(0, T]$

$$
\left\|u^{\prime \prime}-A u\right\|^{2} \leq 2\left\|u^{\prime \prime}\right\|^{2}+2\|A u\|^{2}-2\left(\xi^{\prime}\right)^{2} e^{-2 \xi}\|v\|^{2}+2\left[\int_{0}^{t} e^{-\xi(s)} d s\right]^{2}\|A v\|^{2} .
$$

Using $\|A v\| \leq K$ and substituting for $\xi^{\prime}$ in (3.8), we get

$$
\begin{aligned}
\left\|u^{\prime}-A u\right\|^{2} & \leq 2\left[\frac{\gamma \phi(t)}{8 t^{2}}-\frac{\mathrm{k}^{2}}{\|\mathrm{v}\|^{2}}\right] \mathrm{e}^{-2 \xi}\|\mathrm{v}\|^{2}+2\left[\int_{0}^{\mathrm{t}} \mathrm{e}^{-\xi(\mathrm{s})} \mathrm{ds}\right]^{2} \mathrm{k}^{2} \\
& -(\gamma / 4) \phi(t) \mathrm{t}^{-2} \mathrm{e}^{-2 \xi(\mathrm{t})}\|\mathrm{v}\|^{2}-2 \mathrm{k}^{2}\left\{\mathrm{e}^{-2 \xi(\mathrm{t})}-\left[\int_{0}^{\mathrm{t}} \mathrm{e}^{-\xi(s)} \mathrm{ds}\right]^{2}\right\} . \\
& \leq(\gamma / 4) \phi(t) \mathrm{t}^{-2} \mathrm{e}^{-2 \xi(t)}\|\mathrm{v}\|^{2}
\end{aligned}
$$

where the last inequality is a result of (3.6). We now apply (3.7) to (3.9) to get

$$
\begin{aligned}
\left\|u^{\prime}-A u\right\|^{2} & \leq \gamma \phi(t) t^{-4}\left[\int_{0}^{t} e^{-2 \xi(s)} d s\right]^{2}\|v\|^{2} \\
& =\gamma \phi(t) t^{-4}\|u(t)\|^{2} \leq \gamma \psi_{0}(t) t^{-4}\|u(t)\|^{2} .
\end{aligned}
$$

Hence $u$ is a nontrivial solution of (3.4) (and therefore (1.2)) on $(0, T]$. This completes the proof.

Necessity. Suppose

$$
\int_{0}^{1} \frac{\psi_{0}(t)}{t} d t<\infty \text { and } \int_{0}^{1} \frac{\psi_{1}(t)}{t} d t<\infty .
$$

We define the function $\psi$ (suppressing its dependence on $\alpha$ since $\alpha$ will be chosen to be $1 / 2$ later (in the proof of Lemma 10)) by

$$
\psi(t)=\psi_{0}\left(t^{\alpha}\right)+\psi_{1}\left(t^{\alpha}\right)
$$

where $0<\alpha<1$. Notice that the function $\psi$ inherits the relevant properties of $\psi_{0}$ and $\psi_{1}$ along with one additional property. In particular, $\psi$ satisfies the following:

$$
\psi>0, \psi^{\prime} \geq 0, \psi^{\prime \prime} \leq 0 \quad \text { on }(0, \infty),
$$


and

$$
\int_{0}^{1} \psi(t) / t d t<\infty \quad(\text { as a result of }(3.11)) \text {. }
$$

In addition, the monotonicity of $\psi_{i}$ yields $\psi_{i}(t) \leq \psi_{i}\left(t^{\alpha}\right)$ for $0 \leq t \leq 1$, $i=0,1$, so that, for any interval $\left(0, T_{0}\right], T_{0} \leq 1$, on which (1.2) is satisfied, we get

$$
\left\|u^{\prime \prime}(t)-A(t) u(t)\right\|^{2} \leq \gamma\left[\mu(t)+\int_{0}^{t} \mu(\eta) d \eta\right] \quad 0<t \leq T_{0}
$$

where $\mu(t)=\psi(t)\left[t^{-4}\|u(t)\|^{2}+t^{-2}\left\|u^{\prime}(t)\right\|^{2}\right]$. Also, part (iii) of condition (II) may be restated with $\psi_{0}$ and $\psi_{1}$ replaced with $\psi$. Lastly, and very importantly, as a result of (3.2), we get

$$
t \psi^{\prime}(t) \leq \alpha \psi(t) \text { (i.e., } \psi(t) / t^{\alpha} \text { is nondecreasing.) on }(0, \infty) \text {. }
$$

Hence, using analysis similar to that for getting inequality (2.7), we get

$$
t^{k} \int_{t}^{T} \eta^{-k-1} \psi(\eta) d \eta \leq \psi(t) /(k-\alpha), k>\alpha>0 \text { and } 0<t \leq T .
$$

Before proving necessity, we develop several lemmas.

LEMMA 5. If $u \in C_{\star}([0, T] ; D)$ for some $T>0$ and $u(0)-u^{\prime}(0)=0$, then

$$
\int_{0}^{t} e^{-2 \varphi(s)} s^{-2} \rho(s)\|\mathrm{u}(\mathrm{s})\|^{2} \mathrm{ds} \leq 4(3-\alpha)^{-2} \int_{0}^{t} \mathrm{e}^{-2 \varphi(s)} \rho(\mathrm{s})\left\|\mathrm{u}^{\prime}(\mathrm{s})\right\|^{2} \mathrm{ds}, \quad 0 \leq t \leq \mathrm{T}
$$

where $\rho(t)=\psi(t) / t^{2}, \varphi(t)--\left(t+t_{0}\right)^{-\beta}$ and $t_{0}>0$.

PROOF. Since $u(0)-u^{\prime}(0)-0$, we have $\|u(s)\|^{2}-2 \int_{0}^{s}\left(u, u^{\prime}\right) d \eta \leq 2 \int\|u\|\left\|u^{\prime}\right\| d \eta$. Multiply this inequality by $e^{-2 \varphi} s^{-2} \rho$ and integrate to get

$$
\int_{0}^{t} e^{-2 \varphi} s^{-2} \rho\|\mathrm{u}\|^{2} \mathrm{ds} \leq 2 \int_{0}^{t} \mathrm{e}^{-2 \varphi} \mathrm{s}^{-2} \rho \int_{0}^{\mathrm{s}}\|\mathrm{u}\|\left\|\mathrm{u}^{\prime}\right\| \mathrm{d} \eta \mathrm{d} s--2 \int_{0}^{\mathrm{t}} \mathrm{e}^{-2 \varphi} \Psi^{\prime}(\mathrm{s}) \int_{0}^{\mathrm{s}}\|\mathrm{u}\|\left\|\mathrm{u}^{\prime}\right\| \mathrm{d} \eta \mathrm{d} s
$$

where $\Psi(s)=\int_{s}^{t} \eta^{-2} \rho(\eta)$ d $\eta$ for $0<s \leq t$. Now integrate by parts on the right side of (3.18) to get

$$
\begin{aligned}
& -2 \int_{0}^{t} e^{-2 \varphi_{\Psi}} \int_{0}^{s}\|u\|\left\|u^{\prime}\right\| d \eta d s=\lim _{\varepsilon \downarrow 0}-\left.2 e^{-2 \varphi} \varphi_{0}^{s}\|u\|\left\|u^{\prime}\right\| d \eta\right|_{\varepsilon} ^{t}+2 \int_{0}^{t} \Psi \frac{d}{d s}\left[e^{-2 \varphi} \int_{0}^{s}\|u\|\left\|u^{\prime}\right\| d \eta\right] d s \\
& \leq \lim _{\varepsilon+0} 2 e^{-2 \varphi(\varepsilon)} \Psi(\varepsilon) \int_{0}^{\varepsilon}\|u\|\left\|u^{\prime}\right\| d \eta+2 \int_{0}^{t} \Psi \frac{d}{d s}\left[e^{-2 \varphi} \int_{0}^{s}\|u\|\left\|u^{\prime}\right\| d \eta\right] d s .
\end{aligned}
$$

We now observe that the limit on the right side of (3.19) is zero. To prove this, note that (3.13) implies the existence of a positive constant $C$ (depending on $t$ ) for which $\int_{\varepsilon}^{t} \psi(s) / s$ ds $\leq C$ which yields $\Psi(\varepsilon) \leq \varepsilon^{-3} \int_{\varepsilon}^{t} \psi(s) / s$ ds $\leq C \varepsilon^{-3}$. Now apply L'Hospital's rule to get

$$
\lim _{\varepsilon \downarrow 0} \Psi(\varepsilon) \int_{0}^{\varepsilon}\|\mathrm{u}\|\left\|\mathrm{u}^{\prime}\right\| \mathrm{d} \eta \leq \mathrm{C} \underset{\varepsilon \downarrow 0}{\lim } \varepsilon^{-3} \int_{0}^{\varepsilon}\|\mathrm{u}\|\left\|\mathrm{u}^{\prime}\right\| \mathrm{d} \eta-\lim _{\varepsilon \downarrow 0}-3 \varepsilon^{-2}\|\mathrm{u}(\varepsilon)\|\left\|\mathrm{u}^{\prime}(\varepsilon)\right\|-0
$$


since $u(0)=u^{\prime}(0)-0$ and $u^{\prime \prime}$ is bounded near zero. Thus, after doing the indicated differentiation, inequality (3.19) becomes

$$
\begin{gathered}
-2 \int_{0}^{t} e^{-2 \varphi} \Psi^{\prime} \int_{0}^{s}\|u\|\left\|u^{\prime}\right\| d \eta d s \leq-4 \int_{0}^{t} \Psi \varphi^{\prime} e^{-2 \varphi} \int_{0}^{s}\|u\|\left\|u^{\prime}\right\| d \eta d s+2 \int_{0}^{t} \Psi e^{-2 \varphi}\|u\|\left\|u^{\prime}\right\| d s \\
\leq 2 \int_{0}^{t} \Psi e^{-2 \varphi}\|u\|\left\|u^{\prime}\right\| d s
\end{gathered}
$$

where the last inequality holds since $\varphi^{\prime}>0$. Inequality (3.16) with $k=3$ yields $\Psi(s) \leq$ $s^{-3} \psi(s) /(3-\alpha)=s^{-1} \rho(s) /(3-\alpha)$. Substitution of this into (3.20) and application of Cauchy-Schwarz gives

$$
\begin{aligned}
-2 \int_{0}^{t} e^{-2 \varphi} \Psi^{\prime} \int_{0}^{s}\|u\|\left\|u^{\prime}\right\| d \eta d s & \leq 2(3-\alpha)^{-1} \int_{0}^{t} s^{-1} \rho(s) e^{-2 \varphi}\|u\|\left\|u^{\prime}\right\| d s \\
& \leq 2(3-\alpha)^{-1}\left[\int_{0}^{t} s^{-2} \rho e^{-2 \varphi}\|u\|^{2} d s\right]^{1 / 2}\left[\int_{0}^{t} \rho e^{-2 \varphi}\left\|u^{\prime}\right\|^{2} d s\right]^{1 / 2}
\end{aligned}
$$

Substitution of (3.21) into (3.18) and simplification yields (3.17). This completes the proof.

LEMMA 6. Suppose $z \in C_{*}\left(\left[0, T_{0}\right] ; D\right)$ for some $T_{0}>0$ and $z(0)=z^{\prime}(0)=0$. Then

$$
\int_{0}^{t}\left(\varphi^{\prime}\right)^{2} \rho\|z\|^{2} \mathrm{ds} \leq \lambda\left(\mathrm{T}_{1}\right) \int_{0}^{t}\left\|2 \varphi^{\prime} z^{\prime}+\varphi^{\prime \prime} z-N z\right\|^{2} \mathrm{ds} \quad \text { for any } \mathrm{T} \leq \min \left(\mathrm{T}_{0}, \mathrm{~T}_{1}\right)
$$

where $\varphi$ and $\rho$ are defined as in Lemma 5 and $\lambda(t)=\int_{0}^{t} \psi(s) / s$ ds.

PROOF. Since the function $\lambda$ is increasing, it suffices to prove (3.22) for $T_{1}-$ $t$. The operator $\mathrm{N}$ is antisymmetric and hence $(\eta>0)$

$$
\begin{aligned}
& \operatorname{Re} \int_{0}^{\eta}\left(\varphi^{\prime} z, 2 \varphi^{\prime} z^{\prime}+\varphi^{\prime \prime} z-N z\right) d s=\operatorname{Re} \int_{0}^{\eta}\left[2\left(\varphi^{\prime}\right)^{2}\left(z, z^{\prime}\right)+\varphi^{\prime} \varphi^{\prime \prime}\|z\|^{2}\right] \mathrm{d} s \\
& =\int_{0}^{\eta}\left[\left(\varphi^{\prime}\right)^{2}\|z\|^{2}\right]^{\prime} \mathrm{d} s-\int_{0}^{\eta} \varphi^{\prime} \varphi^{\prime \prime}\|z\|^{2} \mathrm{ds}=\left(\varphi^{\prime}(\eta)\right)^{2}\|z(\eta)\|^{2}-\int_{0}^{\eta} \varphi^{\prime} \varphi^{\prime \prime}\|z\|^{2} \mathrm{ds} z\left(\varphi^{\prime}(\eta)\right)^{2}\|z(\eta)\|^{2}
\end{aligned}
$$

since $\varphi^{\prime} \varphi^{\prime \prime} \leq 0$. Multiply (3.23) by $\rho(\eta)$ and integrate to get

$$
\begin{aligned}
\int_{0}^{t} \rho\left(\varphi^{\prime}\right)^{2}\|\mathrm{z}\|^{2} \mathrm{~d} \eta & \leq \operatorname{Re} \int_{0}^{t} \rho(\eta) \int_{0}^{\eta}\left(\varphi^{\prime} z, 2 \varphi^{\prime} z^{\prime}+\varphi^{n} \mathrm{z}-\mathrm{Nz}\right) \mathrm{d} s \mathrm{~d} \eta \\
& \left.\leq \int_{0}^{\mathrm{t}} \rho(\eta) \int_{0}^{\eta}\left\|\varphi^{\prime} z\right\| \| 2 \varphi^{\prime} \mathrm{z}^{\prime}+\varphi^{n} \mathrm{z}-\mathrm{Nz}\right) \| \mathrm{d} s \mathrm{~d} \eta
\end{aligned}
$$

Application of (2.8) to (3.24) (with $h=\left\|\varphi^{\prime} z\right\|$ and $r-\| 2 \varphi^{\prime} z^{\prime}+\varphi^{n} z-N z$ ) $\|$ ) yields

$$
\int_{0}^{t} \rho\left(\varphi^{\prime}\right)^{2}\|\mathrm{z}\|^{2} \mathrm{~d} \eta \leq(\varepsilon / 2) \int_{0}^{t} \rho\left\|\varphi^{\prime} z\right\|^{2} \mathrm{~d} \eta+(2 \varepsilon)^{-1} \lambda(t) \int_{0}^{t}\left\|2 \varphi^{\prime} z^{\prime}+\varphi^{\prime \prime} z-N z\right\|^{2} \mathrm{~d} \eta .
$$

Putting $\varepsilon=1$ in (3.25) and simplification yields (3.22) for $T_{1}-t$. This completes the proof.

LEMMA 7. Suppose the operator A satisfies condition (II) and $L u=u^{\prime \prime}$ - Au. Let $\varphi$ and $\rho$ be as in Lemma 5 with $t_{0}+T<1$ and suppose $u \epsilon C_{*}([0, T] ; D)$. In addition, assume $u(0)=u^{\prime}(0)=u(T)=u^{\prime}(T)=0$. Then, for $\varepsilon>0$, we get 


$$
\begin{aligned}
\int_{0}^{\mathrm{T}} \rho \mathrm{e}^{-2 \varphi}(\mathrm{Mu}, \mathrm{u}) \mathrm{dt} \leq & {\left[-1+4\left(3+2 \varepsilon+4 \varepsilon^{-1} \psi(\mathrm{T})\right)(3-\alpha)^{-2}\right] \int_{0}^{\mathrm{T}} \rho \mathrm{e}^{-2 \varphi}\left\|\mathrm{u}^{\prime}\right\|^{2} \mathrm{dt} } \\
& +(3 / \varepsilon) \int_{0}^{\mathrm{T}}\left(\varphi^{\prime}\right)^{2} \rho \mathrm{e}^{-2 \varphi}\|\mathrm{u}\|^{2} \mathrm{dt}+\varepsilon \int_{0}^{\mathrm{T}} \mathrm{e}^{-2 \varphi}\|\mathrm{Lu}\|^{2} \mathrm{dt} .
\end{aligned}
$$

PROOF. Using the definition of the operator $L$ and the antisymmetry of $N$, we get (All of the following integrals are taken over $[0, \mathrm{~T}]$. )

$$
\begin{aligned}
\int \rho e^{-2 \varphi}(\mathrm{Mu}, \mathrm{u}) \mathrm{dt} & =\int \rho \mathrm{e}^{-2 \varphi}\left(\mathrm{u}^{\prime \prime}-\mathrm{Lu}-\mathrm{Nu}, \mathrm{u}\right) \mathrm{dt} \\
& =\operatorname{Re} \int \rho \mathrm{e}^{-2 \varphi}\left(\mathrm{u}^{\prime \prime}, \mathrm{u}\right) \mathrm{dt}-\operatorname{Re} \int \rho \mathrm{e}^{-2 \varphi}(\mathrm{Lu}, \mathrm{u}) \mathrm{dt}=\mathrm{J}_{1}+\mathrm{J}_{2} .
\end{aligned}
$$

Integration by parts twice in $J_{1}$ and using the fact that $u$ and $u^{\prime}$ vanish at both 0 and $\mathrm{T}$ yields

$$
\mathrm{J}_{1}=-\int \rho \mathrm{e}^{-2 \varphi}\left\|\mathrm{u}^{\prime}\right\|^{2} \mathrm{dt}+(1 / 2) \int\left(\rho \mathrm{e}^{-2 \varphi}\right) "\|\mathrm{u}\|^{2} \mathrm{dt} .
$$

Since $\left(\rho \mathrm{e}^{-2 \varphi}\right) "=\mathrm{e}^{-2 \varphi} t^{-4}\left(t^{2} \psi^{\prime \prime}-4 t \psi^{\prime}+6 \psi-4 t^{2} \varphi^{\prime} \psi^{\prime}+8 t \varphi^{\prime} \psi+4 t^{2} \psi\left(\varphi^{\prime}\right)^{2}-2 t^{2} \psi \varphi^{\prime \prime}\right), \psi^{\prime} \geq 0, \psi^{\prime \prime} \leq 0$ and $\varphi^{\prime}>0$, we get

$$
\begin{aligned}
\left(\rho \mathrm{e}^{-2 \varphi}\right)^{\prime \prime} & \leq \mathrm{e}^{-2 \varphi}\left(6 \mathrm{t}^{-4} \psi+8 \mathrm{t}^{-3} \varphi^{\prime} \psi+4 t^{-2} \psi\left(\varphi^{\prime}\right)^{2}-2 t^{-2} \psi \varphi^{\prime \prime}\right) \\
& =\mathrm{e}^{-2 \varphi}\left(6 t^{-2} \rho+8 t^{-1} \varphi^{\prime} \rho+4 \rho\left(\varphi^{\prime}\right)^{2}-2 \rho \varphi^{\prime \prime}\right) .
\end{aligned}
$$

Hence substitution of this into (3.28) yields

$$
\mathrm{J}_{1} \leq-\int \rho \mathrm{e}^{-2 \varphi}\left\|\mathrm{u}^{\prime}\right\|^{2} \mathrm{dt}+\int \mathrm{e}^{-2 \varphi}\left(3 \mathrm{t}^{-2} \rho+4 \mathrm{t}^{-1} \varphi^{\prime} \rho+2 \rho\left(\varphi^{\prime}\right)^{2}-\rho \varphi^{\prime \prime}\right)\|\mathrm{u}\|^{2} \mathrm{dt} .
$$

To estimate the right side of (3.29), we observe that $-\varphi^{\prime \prime} \leq 2\left(\varphi^{\prime}\right)^{2}$ for $\beta$ large since $t_{0}+\mathrm{T}<1$, and for $\varepsilon>0$, we get $4 t^{-1} \varphi^{\prime} \rho \leq 2 \varepsilon t^{-2} \rho+2 \varepsilon^{-1} \rho\left(\varphi^{\prime}\right)^{2}$. Applying these two inequalities to $(3.29)$ produces

$$
\mathrm{J}_{1} \leq-\int \rho \mathrm{e}^{-2 \varphi}\left\|\mathrm{u}^{\prime}\right\|^{2} \mathrm{dt}+(3+2 \varepsilon) \int \mathrm{e}^{-2 \varphi} \mathrm{t}^{-2} \rho\|\mathrm{u}\|^{2} \mathrm{dt}+(4+2 / \varepsilon) \int \rho\left(\varphi^{\prime}\right)^{2} \mathrm{e}^{-2 \varphi}\|\mathrm{u}\|^{2} \mathrm{~d} t .
$$

Now apply (3.17) to the second integral on the right side of this inequality to get

$$
\mathrm{J}_{1} \leq\left[-1+4(3+2 \varepsilon)(3-\alpha)^{-2}\right] \int \mathrm{e}^{-2 \varphi} \rho\left\|\mathrm{u}^{\prime}\right\|^{2} \mathrm{dt}+(4+2 / \varepsilon) \int \rho\left(\varphi^{\prime}\right)^{2} \mathrm{e}^{-2 \varphi}\|\mathrm{u}\|^{2} \mathrm{dt} \text {. }
$$

The monotonicity of $\psi$ and application of (3.17) allows the estimate

$$
\begin{aligned}
J_{2} & \leq \varepsilon \int e^{-2 \varphi}\|\mathrm{Lu}\|^{2} \mathrm{dt}+(4 / \varepsilon) \int \mathrm{e}^{-2 \varphi} \rho^{2}\|\mathrm{u}\|^{2} \mathrm{dt} \\
& \leq \varepsilon \int \mathrm{e}^{-2 \varphi}\|\mathrm{Lu}\|^{2} \mathrm{dt}+(4 / \varepsilon) \psi(\mathrm{T}) \int \mathrm{t}^{-2} \rho \mathrm{e}^{-2 \varphi}\|\mathrm{u}\|^{2} \mathrm{dt} \\
& \leq \varepsilon \int \mathrm{e}^{-2 \varphi}\|\mathrm{Lu}\|^{2} \mathrm{dt}+4\left(4 \varepsilon^{-1}(3-\alpha)^{-2} \mid \psi(\mathrm{T}) \int \rho \mathrm{e}^{-2 \varphi}\left\|\mathrm{u}^{\prime}\right\|^{2} \mathrm{~d} t .\right.
\end{aligned}
$$

Substitution of (3.30) and (3.31) into (3.27) gives (3.26) provided $\varepsilon$ is sufficiently small that $4+2 / \varepsilon<3 / \varepsilon$. This completes the proof.

LEMMA 8. Let $z, u, \rho$ and $\varphi$ be as in Lemma 7. Then, for $\varepsilon>0$ small, we get

$$
\int_{0}^{T} \rho\left\|z^{\prime}\right\|^{2} d t \geq\left[1-4 \varepsilon(3-\alpha)^{-2}\right] \int_{0}^{T} \rho e^{-2 \varphi}\left\|u^{\prime}\right\|^{2} d t-2 \varepsilon^{-1} \int_{0}^{T}\left(\varphi^{\prime}\right)^{2} \rho e^{-2 \varphi}\|u\|^{2} d t .
$$

PROOF. Since $z=e^{-2 \varphi} u$, we get (A1l integrals are taken over $[0, T]$. )

$$
\begin{aligned}
\int \rho\left\|z^{\prime}\right\|^{2} d t & =\int \rho e^{-2 \varphi}\left\|u^{\prime}-\varphi^{\prime} u\right\|^{2} d t \\
& =\int \rho e^{-2 \varphi}\left\|u^{\prime}\right\|^{2} d t-2 \operatorname{Re} \int \rho \varphi^{\prime} e^{-2 \varphi}\left(u, u^{\prime}\right) d t+\int \rho\left(\varphi^{\prime}\right)^{2} e^{-2 \varphi}\|u\|^{2} d t .
\end{aligned}
$$


Integrating by parts in the second integral on the right side of (3.33) and using $\varphi^{\prime \prime}$ $\geq-\left(\varphi^{\prime}\right)^{2}$, for $\beta$ large, gives

$$
\begin{aligned}
-2 \operatorname{Re} \int \rho \varphi^{\prime} \mathrm{e}^{-2 \varphi}\left(\mathrm{u}, \mathrm{u}^{\prime}\right) \mathrm{dt} & -\int\left(\rho \varphi^{\prime} \mathrm{e}^{-2 \varphi}\right) \cdot\|\mathrm{u}\|^{2} \mathrm{dt}-\int\left(\rho^{\prime} \varphi^{\prime}+\rho \varphi^{\prime \prime}-2 \rho\left(\varphi^{\prime}\right)^{2}\right) \mathrm{e}^{-2 \varphi}\|\mathrm{u}\|^{2} \mathrm{dt} \\
& \geq \int\left(\rho^{\prime} \varphi^{\prime}-3 \rho\left(\varphi^{\prime}\right)^{2}\right) \mathrm{e}^{-2 \varphi}\|\mathrm{u}\|^{2} \mathrm{dt} .
\end{aligned}
$$

Since $\psi^{\prime} \geq 0$, we get $\rho^{\prime} \geq-2 \rho / t$ and hence $\rho^{\prime} \varphi^{\prime} \geq-2 \rho \varphi / t \geq-\varepsilon \rho / t^{2}-\rho\left(\varphi^{\prime}\right)^{2} / \varepsilon$. Substitute this into (3.34) and that result into (3.33) to get

$$
\int \rho\left\|z^{\prime}\right\|^{2} d t \geq \int \rho e^{-2 \varphi}\left\|u^{\prime}\right\|^{2} d t-\varepsilon \int t^{-2} e^{-2 \varphi} \rho\|u\|^{2} d t-(2+1 / \varepsilon) \int \rho\left(\varphi^{\prime}\right)^{2} e^{-2 \varphi}\|u\|^{2} d t .
$$

Now apply (3.17) to the second integral of the right side of (3.35) and use $2+1 / \varepsilon<$ $2 / \varepsilon$ for small $\varepsilon$, we get (3.32). This completes the proof.

LEMMA 9. Suppose the operator A satisfies condition (II) and $z \in C_{*}([0, T] ; D)$ such that $z(0)=z^{\prime}(0)-z(T)-z^{\prime}(T)=0$. Then, for $T_{0} \geq T$ and $u=e^{-\varphi_{z}^{*}}$, we get

$$
\begin{aligned}
\left(2-\mathrm{c}_{\mathrm{T}}\right) \int_{0}^{\mathrm{T}} \rho \mathrm{e}^{-2 \varphi}\left\|\mathrm{u}^{\prime}\right\|^{2} \mathrm{~d} \eta \leq & \varepsilon^{-1} \lambda\left(\mathrm{T}_{0}\right) \int_{0}^{\mathrm{T}}\left\|z^{\prime \prime}+\left(\varphi^{\prime}\right)^{2} z-M z\right\|^{2} \mathrm{~d} \eta \\
& +(5 / \varepsilon) \int_{0}^{\mathrm{T}}\left(\varphi^{\prime}\right)^{2} \rho \mathrm{e}^{-2 \varphi}\|\mathrm{u}\|^{2} \mathrm{~d} \eta+\varepsilon \int_{0}^{\mathrm{T}} \mathrm{e}^{-2 \varphi}\|\mathrm{Lu}\|^{2} \mathrm{dt} .
\end{aligned}
$$

where $\varepsilon>0, c_{T}-\varepsilon+\gamma_{3}(2-\alpha)(1-\alpha)^{-2} \psi(T)+4\left(3+3 \varepsilon+4 \varepsilon^{-1} \psi(T)\right)(3-\alpha)^{-2}$, the function $\lambda$ is defined in Lemma $6, \rho$ and $\varphi$ are defined in Lemma 5 and the operator $L$ is defined in Lemma 7.

PROOF. Since $z^{\prime}(0)=0$, we get

$$
\begin{aligned}
& 2 \int_{0}^{t}\left\|z^{\prime}\right\|\left\|z^{\prime \prime}+\left(\varphi^{\prime}\right)^{2} z-M z\right\| d s \geq 2 \operatorname{Re} \int_{0}^{t}\left(z^{\prime}, z^{\prime \prime}+\left(\varphi^{\prime}\right)^{2} z-M z\right) d s
\end{aligned}
$$

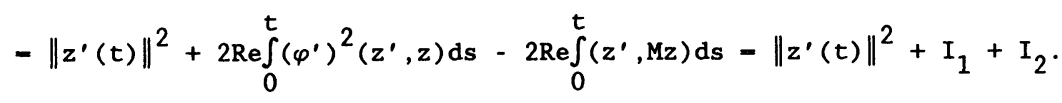

We now estimate $I_{1}$ and $I_{2}$. Integration by parts gives

$$
\begin{aligned}
I_{1} & =\underset{0}{2 \operatorname{Re} \int_{0}^{t}\left(\varphi^{\prime}\right)^{2}\left(z^{\prime}, z\right) d s}=\int_{0}^{t}\left(\varphi^{\prime}\right)^{2}\left(\|z\|^{2}\right)^{\prime} \mathrm{ds} \\
& =\left.\left(\varphi^{\prime}\right)^{2}\|z\|^{2}\right|_{0} ^{t}-\underset{0}{2} \varphi^{\prime} \varphi^{\prime} \varphi^{\prime \prime}\|z\|^{2} \mathrm{ds}=\left(\varphi^{\prime}(t)\right)^{2}\|z(t)\|^{2}-2 \int_{0}^{t} \varphi^{\prime} \varphi^{\prime \prime}\|z\|^{2} \mathrm{ds} \geq 0 .
\end{aligned}
$$

This last inequality is true since $\varphi^{\prime} \varphi^{\prime \prime} \leq 0$. To estimate $I_{2}$, we use (iii) of condition (II) (using $\psi$ in the expression for $F$ instead of $\psi_{0}$ and $\psi_{1}$ ) to get

$$
\begin{aligned}
I_{2} & =-2 \int_{0}^{t}\left(z^{\prime}, M z\right) d s \geq \int_{0}^{t}\left(-F-(M z, z)^{\prime}\right) d s \\
& \geq-\gamma_{3} \int_{0}^{t} \psi(s)\left(s^{-3}\|z\|^{2} d s+s^{-1}\left\|z^{\prime}\right\|^{2}\right) d s-(M(t) z(t), z(t))
\end{aligned}
$$

We now give an estimate for $\int_{0}^{t} s^{-3} \psi(s)\|z\|^{2}$ ds. Since $z(0)=0$, we know 
$\|z(t)\|^{2} \leq t \int_{0}^{t}\left\|z^{\prime}(s)\right\|^{2}$ ds and apply this to get

$$
\int_{0}^{t} s^{-3} \psi(s)\|z\|^{2} \mathrm{ds} \leq \int_{0}^{t} \rho(s) \iint_{0}^{s}\left\|z^{\prime}(\eta)\right\|^{2} \mathrm{~d} \eta \mathrm{d} s \leq-\int_{0}^{t} \frac{\mathrm{d}}{\mathrm{ds}}\left[\int_{s}^{t} \xi^{-2} \psi(\xi) \mathrm{d} \xi\right] \int_{0}^{s}\left\|z^{\prime}(\eta)\right\|^{2} \mathrm{~d} \eta \mathrm{d} s .
$$

Integrating by parts in (3.40) and using (3.16) with $k=1$, we get

$$
\int_{0}^{t} s^{-3} \psi(s)\|z\|^{2} \mathrm{ds} \leq \int_{0}^{t}\left[\int_{s}^{t} \xi^{-2} \psi(\xi) \mathrm{d} \xi\right]\left\|z^{\prime}(s)\right\|^{2} \mathrm{ds} \leq(1-\alpha)^{-1} \int_{0}^{t} s^{-1} \psi(s)\left\|z^{\prime}(s)\right\|^{2} \mathrm{ds} .
$$

Substitution of (3.41) into (3.39) gives

$$
I_{2} \geq-c_{\alpha} \int_{0}^{t} s^{-1} \psi(s)\left\|z^{\prime}(s)\right\|^{2} d s-(M(t) z(t), z(t))
$$

where $c_{\alpha}-\gamma_{3}(2-\alpha) /(1-\alpha)$ and $\alpha$ comes from the definition of $\psi$. Combining (3.37), (3.38) and (3.42), we get

$$
\begin{gathered}
\left\|z^{\prime}(t)\right\|^{2}-c_{\alpha} \int_{0}^{t} s^{-1} \psi(s)\left\|z^{\prime}(s)\right\|^{2} d s-(M(t) z(t), z(t)) \\
\leq 2 \int_{0}^{t}\left\|z^{\prime}\right\|\left\|z^{\prime \prime}+\left(\varphi^{\prime}\right)^{2} z-M z\right\| d s .
\end{gathered}
$$

Multiply (3.43) by $\rho(t)$ and integrate to get

$$
\begin{gathered}
\int_{0}^{T} \rho\left\|z^{\prime}\right\|^{2} d t-c_{\alpha} \int_{0}^{T} \rho(t) \int_{0}^{t} s^{-1} \psi(s)\left\|z^{\prime}(s)\right\|^{2} d s d t-\int_{0}^{T} \rho(M z, z) d t \\
\leq 2 \int_{0}^{T} \rho(t) \int_{0}^{t}\left\|z^{\prime}\right\|\left\|z^{\prime \prime}+\left(\varphi^{\prime}\right)^{2} z-M z\right\| d s d t .
\end{gathered}
$$

To estimate the second integral in (3.44), we let $P(t)-\int_{t}^{T} \rho(\eta) d \eta$ and note that integration by parts produces $\left(h(t)=t^{-1} \psi(t)\left\|z^{\prime}(t)\right\|^{2}\right)$

$$
\begin{aligned}
& \int_{0}^{T} \rho(t) \int_{0}^{t} h(\eta) d \eta d t=-\int_{0}^{T} P^{\prime}(t) \int_{0}^{t} h(\eta) d \eta d t \\
& --P(T) \int_{0}^{T} h(\eta) d \eta+\lim _{\varepsilon \downarrow 0} P(\varepsilon) \int_{0}^{\varepsilon} h(\eta) d \eta+\int_{0}^{T} P(\eta) h(\eta) d \eta .
\end{aligned}
$$

But $\mathrm{P}(\varepsilon) \int_{0}^{\varepsilon} \mathrm{h}(\mathrm{s}) \mathrm{ds} \leq\left[\int_{\varepsilon}^{\mathrm{T}} \mathrm{t}^{-1} \psi(t)\right](1 / \varepsilon) \int_{0}^{\varepsilon} \mathrm{h}(\mathrm{s}) \mathrm{ds}$ and since $\mathrm{z}^{\prime}(0)-0$ (and $\psi(0)-0$ because of (3.13)), we get $\lim _{\varepsilon \downarrow 0}(1 / \varepsilon) \int_{0}^{\varepsilon} h(s) d s-\lim _{\varepsilon \downarrow 0} h(\varepsilon)-0$. Hence $\lim _{\varepsilon \downarrow 0} P(\varepsilon) \int_{0}^{\varepsilon} h(s) d s-0$.

Combining this result with the fact that the first term on the right side of (3.45) is nonpositive, we get

$$
\int_{0}^{\mathrm{T}} \rho(\xi) \int_{0}^{\xi} \mathrm{h}(\eta) \mathrm{d} \eta \mathrm{d} \xi \leq \int_{0}^{\mathrm{T}} \mathrm{P}(\eta) \mathrm{h}(\eta) \mathrm{d} \eta
$$


However, $t^{2} P(t)=t^{2} \int_{t}^{T} \eta^{-2} \psi(\eta) d \eta \leq t \psi(t) /(1-\alpha)$ (We have used (3.16) here with $k-1$ and $0<\alpha<1$ to get the last inequality.) Thus $P(t) \leq(1-\alpha)^{-1} t^{-1} \psi(t)$ and hence substitution of this into (3.46) gives

$$
\int_{0}^{\mathrm{T}} \rho(\xi) \int_{0}^{\xi} \mathrm{h}(\eta) \mathrm{d} \eta \mathrm{d} \xi \leq(1-\alpha)^{-1} \int_{0}^{\mathrm{T}} \eta^{-1} \psi(\eta) \mathrm{h}(\eta) \mathrm{d} \eta
$$

Substituting $h(t)=t^{-1} \psi(t)\left\|z^{\prime}(t)\right\|^{2}$ in (3.47) and using the monotonicity of $\psi$ yields

$$
\int_{0}^{\mathrm{T}} \rho(\xi) \int_{0}^{\xi} \mathrm{h}(\eta) \mathrm{d} \eta \mathrm{d} \xi \leq(1-\alpha)^{-1} \psi(\mathrm{T}) \int_{0}^{\mathrm{T}} \rho\left\|\mathrm{z}^{\prime}\right\| \mathrm{d} \eta
$$

Substitution of this inequality into (3.44) gives

$$
\hat{\mathrm{c}} \int_{0}^{\mathrm{T}} \rho\left\|z^{\prime}\right\|^{2} \mathrm{dt}-\int_{0}^{\mathrm{T}} \rho(\mathrm{Mz}, \mathrm{z}) \mathrm{dt} \leq 2 \int_{0}^{\mathrm{T}} \rho(\mathrm{t}) \int_{0}^{\mathrm{t}}\left\|\mathrm{z}^{\prime}\right\|\left\|\mathrm{z}^{n}+\left(\varphi^{\prime}\right)^{2} \mathrm{z}-\mathrm{Mz}\right\| \mathrm{d} \mathrm{sd} \mathrm{t}
$$

where $\hat{c}-1-(1-\alpha)^{-1} c_{\alpha} \phi(T)$. Application of (2.8) to the right side of (3.45) gives, for $T_{0} \geq T$,

$$
\begin{aligned}
(\hat{c}-\varepsilon) \int_{0}^{T} \rho\left\|z^{\prime}\right\|^{2} d t-\int_{0}^{T} \rho(M z, z) d t & \leq \varepsilon^{-1} \lambda(T) \int_{0}^{T}\left\|z^{n}+\left(\varphi^{\prime}\right)^{2} z-M z\right\|^{2} d t \\
& \leq \varepsilon^{-1} \lambda\left(T_{0}\right) \int_{0}^{T}\left\|z^{n}+\left(\varphi^{\prime}\right)^{2} z-M z\right\|^{2} d t .
\end{aligned}
$$

To complete the proof, we substitute (3.32) and (3.26) into (3.49) and simplify. This completes the proof.

LEMMA 10. Suppose the hypothesis of Lemma 9 holds. Then

$$
\left.\int_{0}^{\mathrm{T}}\left(\varphi^{\prime}\right)^{2} \rho \mathrm{e}^{-2 \varphi}\|\mathrm{u}\|^{2} \mathrm{dt}+\mathrm{C}\left(\mathrm{T}, \mathrm{T}_{0}\right) \int_{0}^{\mathrm{T}} \rho \mathrm{e}^{-2 \varphi}\left\|\mathrm{u}^{\prime}\right\|^{2} \mathrm{dt}\right] \leq \int_{0}^{\mathrm{T}} \mathrm{e}^{-2 \varphi}\|\mathrm{Lu}\|^{2} \mathrm{dt}
$$

where $C\left(T, T_{0}\right)=\left[\lambda\left(T_{0}\right)\right]^{-1}\left[.02-\left(3 \gamma_{3}+23.36\right) \psi(T)\right]$.

PROOF. Since $e^{-\varphi} L u=z^{n}+2 \varphi^{\prime} z^{\prime}+\left(\varphi^{\prime}\right)^{2} z+\varphi^{n} z-M z-N z$, we get (All integrals are taken over $[0, \mathrm{~T}]$.)

$$
\begin{aligned}
& \int e^{-2 \varphi}\|L u\|^{2} d t-\int\left\|z^{\prime \prime}+2 \varphi^{\prime} z^{\prime}+\left(\varphi^{\prime}\right)^{2} z+\varphi^{\prime \prime} z-M z-N z\right\|^{2} d t \\
& \quad-\int\left\|z^{n}+\left(\varphi^{\prime}\right)^{2} z-M z\right\|^{2} d t+2 \operatorname{Re} \int\left(z^{\prime \prime}+\left(\varphi^{\prime}\right)^{2} z-M z, 2 \varphi^{\prime} z^{\prime}+\varphi^{n} z-N z\right)+\int\left\|2 \varphi^{\prime} z^{\prime}+\varphi^{\prime \prime} z-N z\right\|^{2}
\end{aligned}
$$

In [1; pp. 70-72], it is shown (for $\nu_{1}-\nu_{2}-\nu_{3}-0$ ) that

$$
\operatorname{Re} \int\left(z^{\prime \prime}+\left(\varphi^{\prime}\right)^{2} z-M z, 2 \varphi^{\prime} z^{\prime}+\varphi^{\prime \prime} z-N z\right) \geq 0 \text {. }
$$

We now apply this result along with (3.22) and (3.36) to (3.51) to obtain

$$
\begin{aligned}
\left(1+\varepsilon^{2}\left[\lambda\left(\mathrm{T}_{0}\right)\right]^{-1}\right) \int \mathrm{e}^{-2 \varphi}\|\mathrm{Lu}\|^{2} \mathrm{dt} & \geq \varepsilon\left[\lambda\left(\mathrm{T}_{0}\right)\right]^{-1}\left(2-\mathrm{c}_{\mathrm{T}}\right) \int \rho \mathrm{e}^{-2 \varphi}\left\|\mathrm{u}^{\prime}\right\|^{2} \mathrm{dt} \\
& +\left[1 / \lambda\left(\mathrm{T}_{1}\right)-5 / \lambda\left(\mathrm{T}_{0}\right)\right] \int\left(\varphi^{\prime}\right)^{2} \rho \mathrm{e}^{-2 \varphi}\|\mathrm{u}\|^{2} \mathrm{dt}
\end{aligned}
$$

In (3.52), choose $\alpha=1 / 2, \varepsilon-\left[\lambda\left(\mathrm{T}_{0}\right)\right]^{1 / 2}$, and $\mathrm{T}_{1}>0$ sufficiently small that 
$1 / \lambda\left(T_{1}\right)-5 / \lambda\left(T_{0}\right)>2$ so that (3.50) follows after simplification. This completes the proof.

We may now prove necessity. We note that Theorem 4 contains the results of [1; Theorem 3] as a special case.

THEOREM 4. (Necessity) Suppose the operator A satisfies condition (II) and there exists $T \in(0, T]$ such that $u \in C_{\star}([0, T] ; D)$ is a solution of $(1.2)$ on $(0, T]$ with $u(0)=u^{\prime}(0)=0$. If the functions $\psi_{i}, i=0,1$, satisfy $(3.11)$, then $u=0$ on $[0, T]$.

PROOF. Proceeding in the same manner as in the proof of Theorem 2, we again use the function $5 \mathrm{u}, \mathrm{T}^{\prime}$ to be chosen below, and note that inequality (3.50) yields

$$
\left.\beta^{2} \int_{0}^{\mathrm{T}^{\prime}-\varepsilon} \tau^{-2 \beta-2} \mathrm{e}^{2 \tau^{-\beta}} \rho\|\mathrm{u}\|^{2} \mathrm{dt}+\mathrm{C}\left(\mathrm{T}^{\prime}, \mathrm{T}_{0}\right) \int_{0}^{\mathrm{T}} \mathrm{e}^{2 \tau^{-\beta}} \rho\left\|\mathrm{u}^{\prime}\right\|^{2} \mathrm{dt}\right] \leq \int_{0}^{\mathrm{T}} \mathrm{e}^{2 \tau^{-\beta}}\|\mathrm{L}(\zeta \mathrm{u})\|^{2} \mathrm{dt}
$$

Application of inequality (3.14) to the right side of (3.53) gives

$$
\begin{array}{r}
\left.\beta^{2} \int_{0}^{T^{\prime}-\varepsilon} \tau^{-2 \beta-2} e^{2 \tau^{-\beta}} \rho\|\mathrm{u}\|^{2} d t+c\left(T^{\prime}, T_{0}\right) \int_{0}^{T^{\prime}-\varepsilon} e^{2 \tau^{-\beta}} \rho\left\|u^{\prime}\right\|^{2} d t\right] \\
\leq \gamma \int_{0}^{T^{\prime}-\varepsilon} e^{2 \tau^{-\beta}}\left[\mu(t)+\int_{0}^{t} \mu(s) d s\right] d t+\int_{T^{\prime}-\varepsilon}^{T^{\prime}} e^{2 \tau^{-\beta}}\|L(\zeta u)\|^{2} d t .
\end{array}
$$

Using estimates identical to those of $[1$, p.64], inequality (3.54) may be simplified to get rid of the $\int_{0}^{t} \mu(s) d s$ term (and then $\gamma$ is replaced with $2 \gamma$ ). If we then apply inequality (3.17) to the resulting inequality, we get

$$
\begin{gathered}
\left.\beta^{2} \int_{0}^{\mathrm{T}^{\prime}-\varepsilon} \tau^{-2 \beta-2} \mathrm{e}^{2 \tau^{-\beta} \rho\|\mathrm{u}\|^{2} \mathrm{dt}+\mathrm{C}\left(\mathrm{T}^{\prime}, \mathrm{T}_{0}\right)} \int_{0}^{\mathrm{T}^{\prime}-\varepsilon} \mathrm{e}^{2 \tau^{-\beta}} \rho\left\|\mathrm{u}^{\prime}\right\|^{2} \mathrm{dt}\right] \\
\leq 2 \gamma\left[1+4(3-\alpha)^{-2}\right] \int_{0}^{\mathrm{T}^{\prime}-\varepsilon} \mathrm{e}^{2 \tau^{-\beta} \rho\left\|\mathrm{u}^{\prime}\right\|^{2} \mathrm{dt}+\int_{\mathrm{T}^{\prime}-\varepsilon}^{\mathrm{T}^{\prime}} \mathrm{e}^{2 \tau^{-\beta}}\|\mathrm{L}(5 \mathrm{u})\|^{2} \mathrm{dt} .}
\end{gathered}
$$

Thus we choose $T^{\prime} \epsilon(0, T]$ small and $T_{0}=T^{\prime}$ so that $C\left(T^{\prime}, T_{0}\right) \geq 2 \gamma\left[1+4(3-\alpha)^{-2}\right]$ (with $\alpha=1 / 2$ ) so that (3.55) may be simplified to get

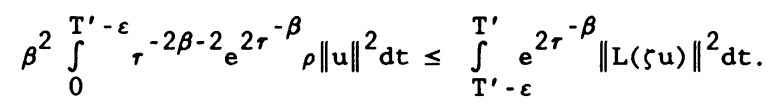

As in $[1, \mathrm{p} .64]$, for $\beta$ large, we may now conclude that

$$
\beta^{2} \int_{0}^{T^{\prime}-\varepsilon} \rho\|\mathrm{u}\|^{2} \mathrm{dt} \leq \int_{\mathrm{T}^{\prime}-\varepsilon}^{\mathrm{T}^{\prime}}\|\mathrm{L}(\zeta \mathrm{u})\|^{2} \mathrm{dt}
$$

Letting $\beta \rightarrow \infty$ we get $u=0$ on $\left[0, T^{\prime}\right]$. This completes the proof.

\section{REFERENCES}

1. HILE, G. N. and PROTTER, M. H. The Cauchy problem and asymptotic decay for solutions of differential inequalities in Hilbert space, Pac. J. Math. 99 (1982), 57-88.

2. LEES, M. and PROTTER, M. H. Unique continuation for parabolic equations and inequalities, Duke Math. J. 28 (1961), 369-382.

3. DONALDSON, J. A. and GOLDSTEIN, J. A. Some remarks on uniqueness for a class of singular abstract Cauchy problems, Proc. Am. Math. Soc. 54 (1976), 149-153. 
4. AMES, K. A. Uniqueness and continuous dependence results for solutions of singular differential equations, J. Math. Anal. Appl. 103 (1984), 172-183.

5. GOLDSTEIN, J.A. The exact amount of nonuniqueness for ordinary differential equations in Banach spaces with an application to the Euler-Poisson-Darboux equation, In Nonlinear Equations in Abstract Spaces (Ed. V. Lakshmikantham) 95-103, Academic Press, New York (1978).

6. ARRATE, M. and GARCIA, A.G. On existence, uniqueness and approximation of solutions of ordinary differential equations in Banach spaces, J. London Math. Soc. 27 (1983), 121-129.

7. GAROFALO, $N$. Uniqueness in the past for parabolic equations with strongly singular potentials, Boll. Un. Mat. Ital. A (6) $\underline{5}$ (1986), 263-271.

8. LAIR, A.V. Uniqueness for singular backward parabolic inequalities, Proc. Am. Math. Soc. 98 (1986), 56-60.

9. SCOTT, J. F. An existence proof for a problem in singular parabolic partial differential equations, J. Inst. Math. Applics. 26 (1980), 221-234.

10. WEINSTEIN, A. Singular partial differential equations and their applications, In Fluid Dynamics and Applied Mathematics (Proc. Sympos., Univ. of Maryland 1961) 29-49, Gordon and Breach, New York, 1962.

11. YOUNG, E. C. Uniqueness of solution of a singular heat equation, Int. J. Math. Math. Sci. I (1984), 201-204.

12. LAIR, A. V. Uniqueness for a nonlinear abstract Cauchy problem, Pac. J. Math. (to appear) 


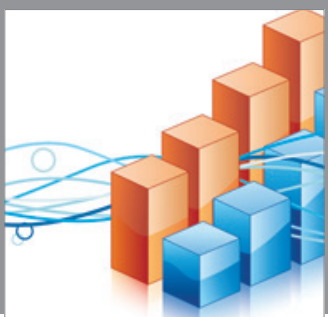

Advances in

Operations Research

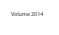

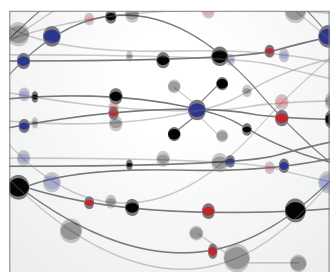

\section{The Scientific} World Journal
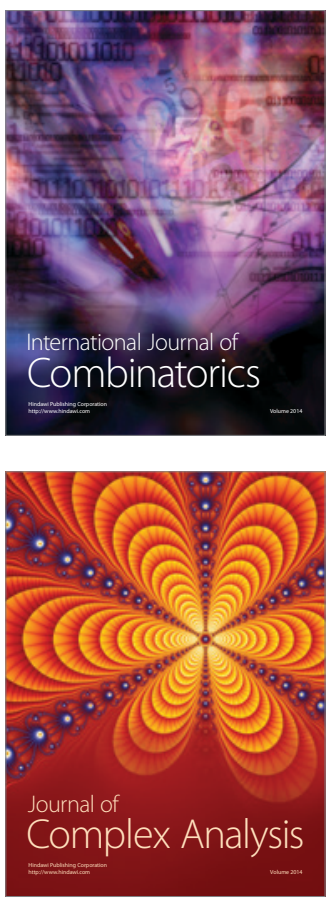

International Journal of

Mathematics and

Mathematical

Sciences
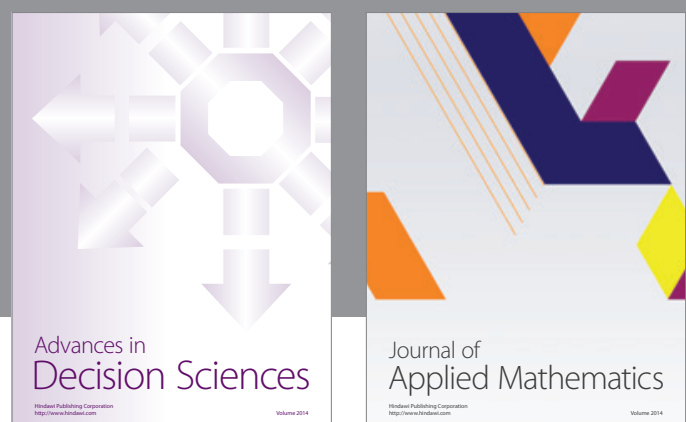

Journal of

Applied Mathematics
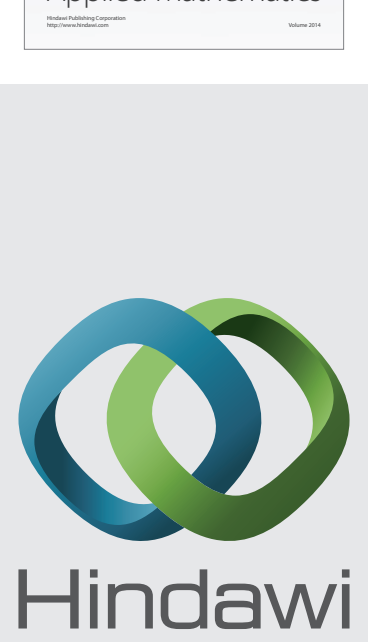

Submit your manuscripts at http://www.hindawi.com
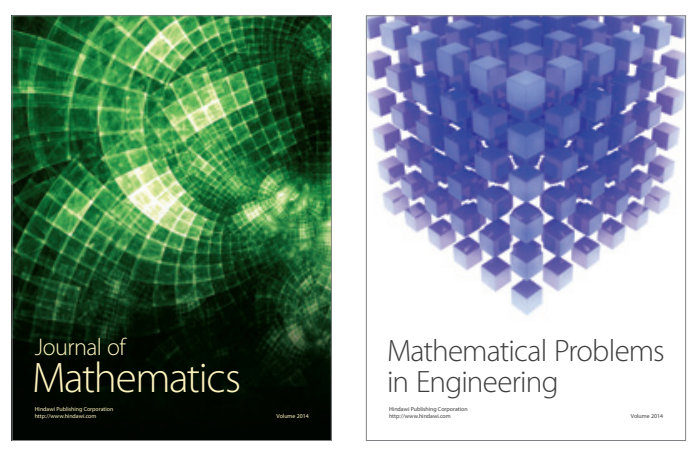

Mathematical Problems in Engineering
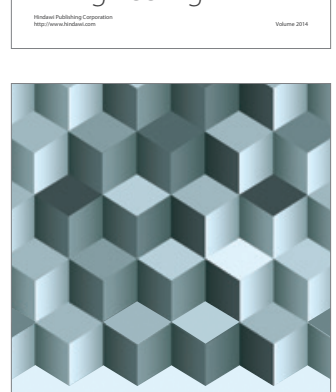

Journal of

Function Spaces
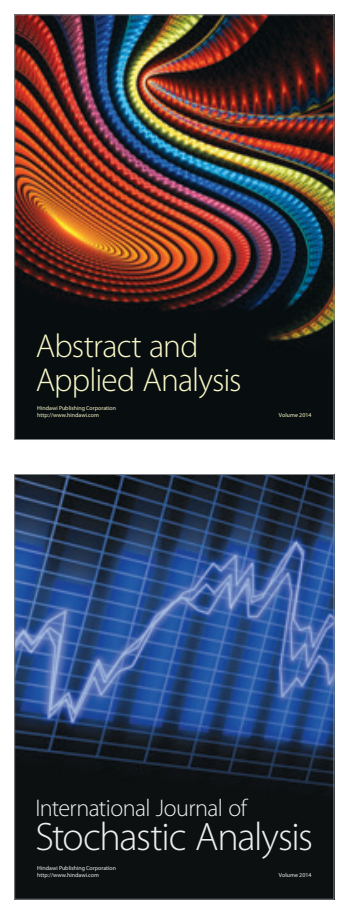

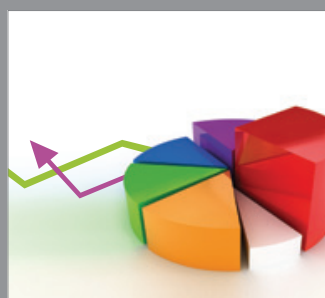

ournal of

Probability and Statistics

Promensencen
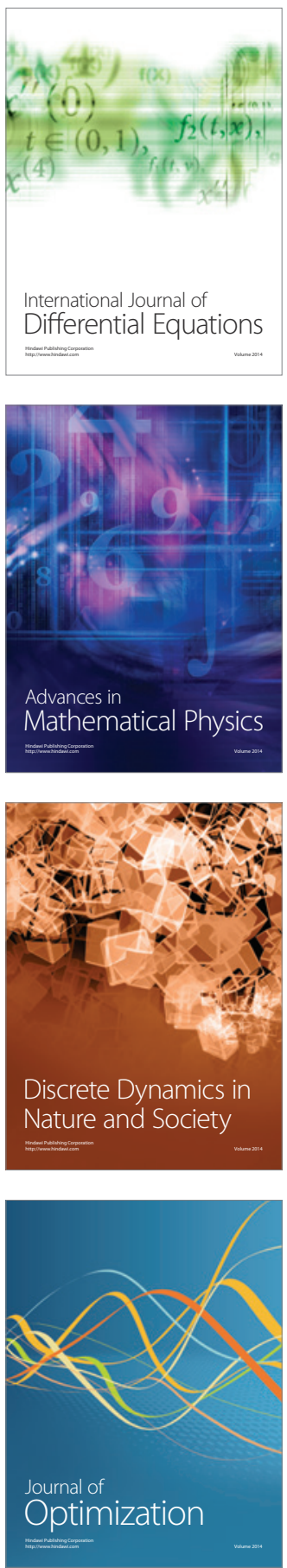